Notfall Rettungsmed 2014 $\cdot 17: 313-313$

DOI 10.1007/s10049-014-1878-z

c) Springer-Verlag Berlin Heidelberg 2014

\author{
J.-T. Gräsner ${ }^{1,2}$ • C. Werner ${ }^{3,4} \cdot$ G. Geldner ${ }^{5,6} \cdot$ B.W. Böttiger ${ }^{7,8}$ \\ ${ }^{1}$ Klinik für Anästhesiologie und Operative Intensivmedizin, \\ Universitätsklinikum Schleswig-Holstein, Campus Kiel \\ ${ }^{2}$ Deutsches Reanimationsregister - German Resuscitation Registry, Nürnberg \\ ${ }^{3}$ Klinik für Anästhesiologie, Universitätsmedizin Mainz \\ ${ }^{4}$ Deutsche Gesellschaft für Anästhesiologie und Operative Intensivmedizin e. V. (DGAl), Nürnberg \\ ${ }^{5}$ Deutsche Gesellschaft für Anästhesiologie und Operative \\ Intensivmedizin e. V. (DGAI), Klinikum Ludwigsburg \\ ${ }^{6}$ Berufsverband Deutscher Anästhesisten e. V. (BDA), Nürnberg \\ ${ }^{7}$ Klinik für Anästhesiologie und Operative Intensivmedizin, Universitätsklinikum Köln \\ ${ }^{8}$ Deutscher Rat für Wiederbelebung e. V., Ulm
}

\title{
10 Thesen für 10.000 Leben
}

\section{Bad Boller Reanimationsgespräche 2014}

75.000-mal im Jahr reanimieren Notarztund Rettungsdienst jedes Jahr in Deutschland. 75.000 Patienten, die nach einem plötzlichen Herztod eine professionelle Behandlung erhalten, um diese ins Leben zurückzubringen. Um dies zu erreichen, ist eine perfekte Behandlungskette von Beginn an notwendig. Hier greifen alle Glieder ineinander, und nur wenn alle diese Abschnitte optimal gestaltet sind, hat ein Patient die Chance zum Überleben. Wir leisten in Deutschland gute Arbeit. Dennoch geht noch mehr! Aktuelle Zahlen aus dem Deutschen Reanimationsregister, insbesondere aber der Vergleich mit den Ergebnissen aus Skandinavien und den Niederlanden, weisen auf ein Optimierungspotenzial in Deutschland hin.

Die Deutsche Gesellschaft für Anästhesiologie und Intensivmedizin e. V. (DGAI), der Berufsverband Deutscher Anästhesisten e. V. (BDA), der Deutsche Rat für Wiederbelebung e.V. (GRC) und das Deutsche Reanimationsregister haben 2014 die Bad Boller Reanimationsgespräche ins Leben gerufen, um diese Optimierung in Deutschland zu erreichen:

Ein Leben zu retten, rechtfertigt alle Bemühungen.

Mehrere Leben zu retten ist ein Privileg.
Gemeinsam, intensiv und konstruktiv diskutierten in Bad Boll 52 Experten aus allen mit dem Thema „Reanimation“ befassten Berufs- und Interessensgruppen. Zum ersten Mal in Deutschland kamen Ärztliche Leiter Rettungsdienst, Vertreter der Kostenträger, Anästhesisten, Chirurgen, Kardiologen, Vertreter von Rettungsdienstorganisationen, Industrie, der Bundesarbeitsgemeinschaft Erste Hilfe und weiteren Verbänden (DIVI, Bundesverband der ÄLRD in Deutschland, Deutsche Herzstiftung, Arbeitsgemeinschaft der Berufsfeuerwehren) zusammen, um die Versorgung der Bevölkerung in Deutschland bei Herz-Kreislauf-Stillstand zu optimieren.

In Bad Boll wurden 10 Thesen für 10.000 Überlebende nach präklinischem Herz-Kreislauf-Stillstand formuliert (http://www.bad-boller-reanimationsgespraeche.de).

In dieser Ausgabe von Notfall + Rettungsmedizin werden diese 10 Thesen einzeln vorgestellt. Jetzt liegt es an uns allen, diese Thesen in die tägliche Versorgung zu überführen.

Wir laden Sie ein, in die eigenen Versorgungs- und Zuständigkeitsbereiche $\mathrm{zu}$ schauen, ihre Prozesse und Strukturen zu überprüfen und mit der Optimierung zu beginnen.

Jede einzelne Verbesserung in einem Teilprozess kann einen Überlebenden mehr bedeuten.

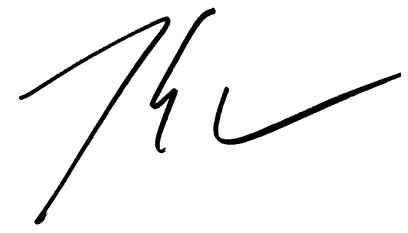

Jan-Thorsten Gräsner

\section{Korrespondenzadresse}

PD Dr. J.-T. Gräsner

Klinik für Anästhesiologie und Operative Intensivmedizin, Universitätsklinikum

Schleswig-Holstein, Campus Kiel

Schwanenweg 21, 24105 Kiel

graesner@reanimationsregister.de

\section{Einhaltung der ethischen Richtlinien}

Interessenkonflikt. J.-T. Gräsner, C. Werner, G. Geldner und B.W. Böttiger geben an, dass kein Interessenkonflikt besteht.

Dieser Beitrag beinhaltet keine Studien an Menschen oder Tieren. 